\title{
Enriching the results of screening Social Life Cycle Assessment using content analysis: A case study of sugarcane in Brazil
}

\author{
Chongyang Du, Cássia Ugaya, Fausto Freire, Luis C. Dias, Roland Clift
}

C. Du; F. Freire (corresponding author)

ADAI-LAETA, Department of Mechanical Engineering, University of Coimbra

R. Luis Reis Santos, 3030-788, Coimbra, Portugal

email: fausto.freire@dem.uc.pt; telephone: + 351239790 739; fax: +351 239790701

C. Ugaya

Post-Graduation Program of Materials and Mechanical Engineering, Federal University of Technology - Paraná, CNPq Fellow

L. C. Dias

INESC Coimbra, CeBER and Faculty of Economics, University of Coimbra

Av. Dias da Silva, 165, 3004-512, Coimbra, Portugal

R. Clift

Centre for Environment and Sustainability, University of Surrey, Guildford GU2 7XH, United Kingdom 
Abstract

Purpose: Screening Social Life Cycle Assessment (S-LCA) can be applied to identify the social hotspots associated with a production activity or supply chain. The objective of this paper is to explore how the quality of the results of a screening S-LCA can be improved, illustrated by a case study of sugarcane production in Brazil, an activity which has been criticized for its records on social sustainability due to issues such as poor working conditions for field workers and treatment of migrant workers.

Methods: Cradle-to-gate production of sugarcane in Brazil has been modelled using input-output analysis. The associated social impacts have been modelled using the framework of the Social Hotspots Database (SHDB), which is one of the first databases providing information on social risks along supply chains. The results from the SHDB were complemented with results from a systematic analysis of relevant literature. Content analysis was applied to 38 publications in English relevant to the social impacts of sugarcane production in Brazil, including peer-reviewed articles, "grey literature", Non-Governmental Organization reports and conference presentations. Qualitative data analysis software NVivo 8 was used to facilitate the analysis of the publications. A deductive category system was established based on the subcategories recommended in the UNEP/SETAC Social Life Cycle Assessment guidelines. Social impacts were further aggregated and analyzed by social themes and impact categories.

Results and discussion: The social impacts of the sugarcane life-cycle in Brazil arise almost exclusively within the Brazilian sugarcane sector itself. Fifteen social themes are identified as hotspots in the SHDB, and nine of them are also identified by content analysis. Health and safety, and labour rights and decent work are the impact categories with the highest risks. Besides negative impacts, content analysis is capable of identifying several positive impacts related to sugarcane production. Due to the use of aggregated country-level data, social impacts of manual and mechanical harvesting of sugarcane cannot be differentiated in SHDB; however, this can be achieved by content analysis.

Conclusions: SHDB is effective for identifying social impacts at the country level but the data are aggregated and only show averages across different technologies and geographical areas; therefore, the database is of limited value in distinguishing between alternative operations. Content analysis can facilitate foreground data collection by differentiating operations and identifying both negative and positive impacts at the activity level. We recommend that S-LCA databases can be integrated with results of content analysis to improve the quality of results from a screening S-LCA and to differentiate between alternative production routes.

Key words: Social life cycle assessment (S-LCA), social impacts, content analysis, Social Hotspots Database (SHDB), sustainable production 


\section{Introduction}

Brazil is the world's largest sugarcane producer, accounting for approximately $36 \%$ of the global production in 2015 (FAO 2016). In 2016, 652 million tonnes of sugarcane were harvested in Brazil, resulting in 39 million tonnes of sugar and 27 billion liters of ethanol produced (UNICA 2017). Awareness about unsustainable production of sugarcane has been raised due to environmental and health concerns associated with pre-harvest burning practices in manual harvesting of sugarcane (Du et al. 2017). São Paulo is the state with the largest sugarcane production in Brazil, accounting for 55\% of national production in 2016 (UNICA 2017). The São Paulo state government and the industrial association have signed a "Green Protocol" to phase out pre-harvest burning by 2017. Because manual harvesting without burning has low productivity, this is leading to increased use of mechanical harvesting (Chaddad 2010): the proportion of sugarcane harvested mechanically without pre-harvest burning in São Paulo has risen from 31\% in 2006 to $89 \%$ in 2014 (CTC 2014).

At the same time, sugarcane producers are under increased pressure to assess and report their social impacts. The most widely used sustainability reporting and certificate schemes, such as Global Reporting Initiative (GRI) and sustainable sugarcane certificate e.g. BONSUCRO (UNICA 2010; BONSUCRO 2017; GRI 2017), use social indicators focused on workers, leaving out other stakeholders. Similarly, publications documenting the social impacts of sugarcane production in Brazil generally concentrate on worker-related topics, covering working conditions, working hours and occupational health and safety (Rocha et al. 2010; Junior et al. 2012; Luz et al. 2012; Souza et al. 2016). Most existing studies are restricted to specific activities in sugarcane production such as planting and/or harvesting, but Souza et al. (2016) investigated the social impacts related to the life-cycles of first and second generation sugarcane ethanol in Brazil; economic input-output models were used, but only inventory indicators within the Brazilian economy were included. Souza et al. (2016) concluded that agricultural operations dominate the impacts of sugarcane ethanol, regardless of the ethanol production technology adopted, because it is by far the most labour-intensive activity in the supply chain. However, a study focusing on the social impacts of sugarcane production in Brazil considering all relevant stakeholders and covering the full life-cycle is lacking.

Social Life Cycle Assessment (S-LCA) is an emerging method to evaluate social impacts related to supply chains (Petti L 2018). It is derived from the well-established environmental assessment method of Life Cycle Assessment (LCA). Compared to other tools assessing social impacts, such as Social Accounting 8000, AccountAbility's AA1000 series, Social Impact Assessment and GRI, S-LCA focuses on a product (or service) level, and considers the entire life-cycle and a broader range of stakeholders, including workers, local community, society, consumers and value chain actors (UNEP/SETAC 2009). Depending on the goal and scope of the study, an S-LCA study can be based on generic and/or site-specific data. Generic data are not site or enterprise specific, and may be collected through literature review, web search or national statistics. Site-specific data can be gathered through document auditing (i.e. enterprise, authorities and Non-Governmental Organization documentations), interviews, 
questionnaires, and participatory evaluation. Generic assessment is appropriate when the aim is to analyze a generic product or to screen social hotspots (i.e. unit processes located in a specified region where a social theme of interest may be considered a problem, a risk or an opportunity). If practitioners need to evaluate the social impacts related to a specific product, site-specific data should be collected for the unit processes considered as social hotspots, but generic data can be used to guide data collection (see below). A further difference between S-LCA and LCA is the treatment of positive impacts. Whereas hotspot assessment follows LCA in focusing on potential negative impacts associated with supply chains, a more complete S-LCA aspires to include both positive and negative impacts (Di Cesare et al. 2018). However, there is no unified definition of positive impacts in S-LCA so far. In the UNEP/SETAC Guidelines for S-LCA (2009), positive impacts are defined as performances beyond compliance with relevant laws, international agreements and certification standards; this approach is consistent with that embodied in the UN's Sustainable Development Goals (Russell et al. 2018). Some researchers have considered positive impacts as the absence of negative issues. For instance, in Ciroth and Franze (2011), one example of positive impacts is the absence of forced labour. Ekener et al. (2016) suggested that reference points for assessing positive impacts should depend on the goal and the scope of the study, and for a case-specific assessment, the regional general behavior can be considered as the reference so that a product system that is more socially beneficial than the local average is considered to have positive impacts. The approach taken in this work is based on the UNEP/SETAC Guidelines, as interpreted by Ekener et al. (2016); see section 2.3.

Data availability is recognized as a critical factor for the development of S-LCA (Benoit-Norris et al. 2013). A typical product system can contain over a thousand unit processes; thus it is not practical to collect site-specific data at every organization along a supply chain, especially considering the increasing globalization of supply chains (Benoit-Norris et al. 2012). Application of a database can ease the burdens of data collection in S-LCA significantly by revealing where in the supply chain attention should be focused. The Social Hotspots Database (SHDB) is one of the first databases in S-LCA which can be utilized as a screening or prioritization tool. The SHDB models the product life-cycle based on global economic input-output data, to identify social risks or opportunities along the supply chains and the unit processes (i.e. country-specific-sectors in SHDB) where site-specific data need to be collected. Data in SHDB are collected at country-sector level; however, due to the aggregation of the data, SHDB is not suitable to differentiate the social impacts of homogeneous sectors (for instance, chemicals, plastics and rubber are aggregated into the same sector in SHDB) or different technologies in the same sector. Arcese et al. (2018) have pointed out the potential of Automated Text Analysis to track the development of concerns and research priorities in S-LCA. The research summarized in this paper takes a different approach, set out in section 2.2, using textual analysis to assess social impacts within a defined product system. Once the social hotspots have been identified using tools like SHDB, publications related to the social impacts of the key country-sector(s) are systematically analysed. The most relevant social themes are identified, covering both negative and positive impacts and differentiating between technologies and approaches to production. The potential of this approach to improve the quality of the results of a screening S-LCA study beyond what is achievable with a Social Hotspots Database is explored using a case study of sugarcane production in Brazil. 
Materials and methods adopted in this study are presented in Section 2. Results are presented in Section 3, and discussed in Section 4.

2. Materials and methods

The Social Hotspots Database (SHDB), with its inbuilt input-output model, was used to carry out a screening S-LCA of cradle-to-gate production of sugarcane in Brazil to identify the associated social hotspots. The default functional unit in SHDB is sugarcane with a value of one USD produced in Brazil; however, the choice of functional unit is irrelevant because the overall results are expressed as dimensionless indices in SHDB, as shown in Section 2.1 below. In the SHDB, the sugarcane product system was represented by an economic input-output model derived from the Global Trade Analysis Project (GTAP) (2017). The level of aggregation and the use of economic values in the model mean that low-value co-produced wastes and residues cannot be included explicitly in the assessment. In effect, it is assumed that waste generated during harvesting remains in the fields (where it may be burned), although some of the tops and leaves of the cane are now brought out for processing into cellulose products. Waste from subsequent processing - primarily bagasse - leaves the system analysed through the farm gate with the cane product.

All the country-sectors providing inputs to the Brazilian sugarcane sector were scaled according to the economic value of their inputs. The impacts of these country-sectors were characterized considering both the risk levels on each social theme and their contributions in terms of labour intensity, estimated using the worker-hour model incorporated in the SHDB. A cut-off criterion based on worker-hours was applied to determine which country-sectors should be included in the system. This analysis confirmed that, overridingly, the social impacts arise within the Brazilian sugarcane sector itself. The dominant country-sector identified in this way was then examined in depth by applying content analysis to the relevant literature.

\subsection{Characterization of social impacts in Social Hotspots Database (SHDB)}

The Social Hotspots Database (SHDB) comprises three components: social theme tables, input-output model and worker-hour model (Benoit-Norris et al. 2012; Benoit-Norris et al. 2013). As shown in Table 1, SHDB groups social indicators into five categories, namely Labour rights and decent work, Health and safety, Human rights, Governance, and Community infrastructure. Each category covers a range of relevant social themes, with one or more indicators to measure the risk level of each theme for a country-sector, including 22 themes and 124 indicators in total. The assessment framework of SHDB for a country-sector from impact category to inventory indicator is shown in Fig. 1. Four risk levels are defined (low, medium, high, and very high) for each indicator of a country-sector. For each theme, the risk level is defined with reference to the range of values reported for the countries included in the database. For instance, for the poverty indicator, percentage of people living under $2 \$ /$ day, the characterization rule of $<2 \%=$ low risk, $2-10 \%=$ medium risk, $10-15 \%=$ high risk, $>50 \%=$ very high risk is used. In the absence of further information, the same risk levels have been used here. For some indicators, such as forced labour, the risk level is determined by whether there is evidence of forced labour and the number of sources of that 
evidence. This approach has been developed further in this work by using systematic content analysis, as set out in Section 2.2.

The SHDB models product supply chains using a global input-output model, which covers economic data of 227 countries and 57 sectors. The economic data for a country-sector is then translated into its labour intensity through a worker-hour model. The total worker-hours of a country-sector are calculated by dividing the total payment of wages to workers (using data from the GTAP model) with wage rate data for that country-sector. An initial analysis using SHDB showed that more than a thousand country-sectors in total are related to sugarcane production in Brazil. As worker-hours represent the level of labour involvement of a country-sector in a supply chain, worker-hours can also be used to set cut-off criterion to exclude country-sectors with little relevance to the product system (Ramirez et al. 2016). An initial cut-off criterion was defined to include only country-sectors contributing more than $1 \%$ of the total worker-hours associated with sugarcane production in Brazil; five sectors met this criterion. The list of sectors to be included was subsequently refined further; see section 3.1.

In the SHDB approach (Benoit-Norris et al. 2012), the scores for the different social themes within each social category are aggregated into a Social Hotspots Index (SHI), defined by Equation 1. The risk levels (R) are translated into indices using the values: low risk $=0$, medium risk $=1$, high risk $=2$, and very high risk $=3$. Equal weights have been assigned to the social themes in several S-LCA case studies (Garrido et al. 2016), and this approach was adopted in this study due to the lack of information on value choices of decision makers. SHI is unitless and its value varies from 0 to 1 . Regardless of the number of indicators in an impact category, the larger the value of SHI, the higher are the potential impacts in that category for a country-sector.

$$
\text { SHIcat }=\sum_{T=1}^{n}(\operatorname{Ravg} \times W T) / \sum_{T=1}^{n}(\operatorname{Rmax} \times W T) \quad \text { Equation } 1
$$

SHIcat: Social Hotspots Index for a category (e.g. labour rights, governance, etc.)

T: Social themes (e.g. child labour, freedom of association rights, etc.

$\mathrm{n}$ : Number of themes within a category

Ravg: Average risk across the theme

Rmax: Maximum risk for a theme

WT: Weight assigned to the theme

In this work, Impact Scores (IS) were also developed to aggregate the social impacts within each category for each of the country-sectors included in the product system. As shown in Equation 2, a weighted sum approach was employed, considering both the overall risk levels and the contribution to labor intensity of a country-sector. Impact Score is a unitless index varying from 0 to 1 , with higher values representing higher potential impacts in the category.

$$
I S=\sum_{m=1}^{K} \text { SHIcat } \times W H \% \quad \text { Equation } 2
$$

IS: Impact Score; overall impacts on an impact category considering all the country-sectors m: a country-sector

$\mathrm{K}$ : Number of country-sectors included in the system boundary

WH\%: Percentage of worker hours out of total worker hours for each country-sector 


\subsection{Content analysis}

To enrich the results of the screening S-LCA using a generic database, content analysis was applied to identify the social impacts of sugarcane production in Brazil by analyzing relevant publications. Content analysis refers to a family of approaches or techniques for studying and/or retrieving meaningful information from text(s) in a systematic manner based on explicit rules of coding (Stemler 2001; Zhang and Wildemuth 2009). The development of content analysis in the scientific arena can be dated to 1920s/30s, with an initial emphasis on quantitative textual analysis such as counting explicit text elements. However, this quantitative approach has been criticized for oversimplified or distorted quantification due to, for instance, inability to consider the cultural components of the context, multiple meanings of words, and multiple expressions for the same meaning. Qualitative content analysis has been developed to overcome these concerns: beyond merely counting words, it emphasizes an integrated view of texts and their specific contexts, and enables subjective but scientific and reproducible inferences to be drawn (Zhang and Wildemuth 2009; Mayring 2014). Quantitative and qualitative content analysis can be combined (Stemler and Bebell 1999), and this combined approach has been applied here: frequency analysis was conducted and reported, and a careful hand-coding of the content of the literature was carried out based on the set of categories identified. The common steps in content analysis were followed; more details on the methodology can be found in Stemler (2001). The steps implemented were:

Step 1: Formulation of issue or problem. This analysis defines the objectives of the work by determining which themes are most documented in recent publications relevant to the social impacts of sugarcane production in Brazil.

Step 2: Selection of the material to be analyzed. Web-based research was used to identify relevant documents by searching Web of Science, Google and Google scholar using the keywords "social impacts", "social sustainability", "corporate social responsibility", "sugarcane", and "Brazil". Documents published in English between 2000 and 2016 were included in the search. In total, 38 articles were considered relevant for content analysis: 21 journal articles, 7 "grey" papers and reports, 7 conference presentations, 2 NGO reports and 1 book chapter.

Step 3: Establishment of a set of categories. Having identified the texts to be searched, the set of categories which the content analysis is to find can be generated inductively (i.e. categories emerge from the material samples) or deductively (i.e. categories are predefined based on social theories or social findings). The set of categories used in this work, shown in Table 2, was established deductively based on the social themes recommended in the UNEP/SETAC Guidelines.

Step 4: Definition of categories and analysis units. Each category was further divided into social themes, defined in accordance with the approach adopted in SHDB (Benoit-Norris et al. 2013; UNEP/SETAC 2013). These social themes were used in step 5 to select the "coding units", i.e. the sections of text to be examined by content analysis in 
step 6. Using themes to select coding units emphasizes the expression of an idea (e.g. the concept of fair salary) rather than the occurrence of the exact words (e.g. "fair salary" or its synonyms) (Zhang and Wildemuth 2009).

Step 5: Coding. The material samples selected in step 2 were hand-coded in the software NVivo 8 to select all coding units alluding to one or more of the themes defined in step 4 (NVivo 2017).

Step 6: Analyzing the coded data. The set of coding units extracted from the samples was analyzed to identify the social themes referred to most frequently in the samples. The frequencies of the social themes within each category were aggregated to give the total frequencies of the categories.

Step 7: Reporting on the findings. Key findings on each social theme were summarized and reported in a descriptive paragraph with identification of key references; these results are discussed in Section 3.2.

\subsection{Defining social hotspots}

There is a lack of consensus in the S-LCA community on the methodology of defining social hotspots. As mentioned in the Introduction, social hotspots are unit processes located in a region where a social theme of interest may be considered a problem, a risk or an opportunity (UNEP/SETAC 2009). Social hotspots can potentially have negative (e.g. problem or risk) or positive (e.g. opportunity) impacts. Following an approach adopted in published studies (Ekener-Petersen et al. 2014; Zamani et al. 2016), the social themes giving rise to the greatest concerns, i.e. the social hotspots indicated by the SHDB, were identified by summing the numbers of indicators with high and very high risks. This approach only considers negative impacts related to the social themes. A similar approach was adopted for the content analysis but accounting for both negative and positive impacts: the social impact themes arising most frequently in the coded samples were considered the social hotspots. Negative and positive impacts were treated equally in the steps of coding and frequency analysis (i.e. Steps 5 and 6 in Section 2.2), and were further analyzed and differentiated by theme in the qualitative analysis of the coded text (i.e. Step 7 in Section 2.2). In this article, positive impacts were considered as performances beyond compliance, such as with laws and international agreements or performances better than the country-sectoral general behaviors. It should be noted that this simple approach of counting indicators or themes may bias the identification of hotspots towards categories with a higher number of indicators. The Social Hotspots Index (SHI), introduced in Section 2.1, is defined to avoid this bias.

\section{Results}

3.1 Life-cycle social impacts of sugarcane production in Brazil and social hotspots identified from SHDB

As stated in Section 2.1, an initial cut-off criterion of including only country-sectors contributing more than $1 \%$ of the total worker-hours associated with sugarcane life-cycle was applied. Table 3 shows the country-sectors remaining after applying this cut-off criterion. The two remaining sectors contributing least to the social impacts of sugarcane life-cycle are "bovine cattle, sheep and goats \& horses" and "animal products" in Brazil. Because SHDB is based on the economic input-output model of GTAP, the model in SHDB takes one USD of Brazilian sugarcane 
output to be associated with inputs of 0.017 USD of animal products and 0.011 USD of bovine cattle, sheep and goats, \& horses in Brazil. It is not clear what connects these two sectors with the Brazilian sugarcane sector. The connection may be indirect, via first-tier suppliers to the sugarcane sector. In view of the lack of transparency over the relationship with these two sectors and their relatively small contributions to the total worker-hours ( $1.2 \%$ each), the cut-off criterion was revised to $1.5 \%$. This leaves only the Brazilian sugarcane, commerce and business service sectors as relevant to the Brazilian sugarcane life-cycle.

Table 4 presents the values of the Social Hotspots Index (SHI) for the five impact categories for these three connected sectors. Health and safety and labour rights and decent work have higher potential social impacts compared with the other impact categories. Within each impact category the value of the SHI is similar (or even the same) across the three sectors, because they are all located in Brazil and the SHDB uses social data at the country level when data at the sector level are not available (Benoit-Norris et al. 2013): close examination of the SHDB handbook revealed that, of 124 indicators, only 18 are based on data at the sector level.

Fig. 2 shows the Impact Scores of the sugarcane life-cycle, aggregated across the country-sectors considering risk levels and contribution to labour intensity according to Equation 2. The sugarcane sector in Brazil is the dominant contributor to social impacts due to its dominance in labour intensity.

Using the approach to determining social hotspots described in Section 2.3, Table 5 shows the indicators with high and very high risks in the sugarcane sector in Brazil, whilst Table 6 ranks them to show the principal Social Hotspots. In total, 37 indicators are identified with high or very high risks related to 15 social themes. "Occupational toxics \& hazards" and "human health due to communicable diseases" are the social themes with the greatest concerns, followed by "high conflict zones" and "migrant workers". Among the 15 social hotspots identified by SHDB, nine are also identified by content analysis, as discussed in the next section.

\subsection{Social hotspots identified by content analysis}

Content analysis identified in total 22 social themes in the text samples examined. The themes mentioned most frequently (coding frequency $>10$ times), i.e. social hotspots, are shown in Fig. 3. By impact category, social themes related to labour rights and decent work arise most frequently in the coded texts, followed by health and safety. Nine of the themes emerge as social hotspots from both the content analysis and the SHDB. "Local employment" emerges as a social hotspot from the content analysis, but not from the SHDB because the database only includes data aggregated at the country level and characterized as medium risk. "Public commitment to sustainability issues" and "contribution to economic development" are identified as social hotspots in content analysis, but these two social themes are not included in SHDB.

Table 7 presents the key findings for each social hotspot based on content analysis. The results of content analysis suggest that the sugarcane sector in Brazil is well-regulated with active collaborations between governments and the industry association, focused on reducing environmental impacts through eliminating pre- 
harvest burning and improving the working conditions of sugarcane field workers. Despite the positive overview at the sectoral level, social impacts of different organizations vary due to their different conducts. For instance, for the social hotspots of "social benefits and social security", "access to material resources" and "freedom of association and collective bargaining", evidences of both positive and negative conducts are identified. Moreover, although in S-LCA good management is often considered as evidence of lower impact (Dreyer et al. 2006; Ramirez et al 2016), the findings on "occupational health and safety" run counter to this assumption: even if adequate protective equipment is provided to manual sugarcane cutters, the nature of the job may still put a heavy toll on workers' longterm health and safety.

The results of content analysis also shed light on the important differences in social impacts between different operations within the sugarcane sector. Harvesting is identified as the most labour-intensive process. The transition from manual to mechanical harvesting, which is especially rapid in the Centre-South region of Brazil, changes the impacts associated with each social hotspot. Fig. 4 compares manual and mechanical harvesting on the social themes where their social impacts differ. Mechanical harvesting has lower impacts in most social themes except for "local employment" and "access to material resources", illustrating the widespread tension between labour intensity and machine use. For manual harvesting, the social theme with the highest potential impact is "occupational health and safety". Exhaustion, back pain, occupational injuries due to fatigue, and high psychological stress have been reported among sugarcane cutters (Rocha et al. 2010; Priuli et al. 2014). This results from the pressure on sugarcane cutters to achieve high productivity: productivity of sugarcane cutters has increased from 6 tonnes/day to 12 tonnes/day in the past decades in order to be competitive with the productivity of mechanical harvesting. High risk associated with a fair salary for manual harvesters also contributes to the concern over health and safety: manual sugarcane cutters are usually paid by productivity rather than a fixed wage and this often motivates them to work beyond their physical limits. The high impacts of delocalization and migration of manual harvesters are related to the evidence of lacking decent living conditions, sanitation and nutritionally adequate food for seasonal migrant workers (Luz et al. 2012).

Compared to manual harvesting, mechanical harvesting has both negative and positive impacts on local employment. One mechanical harvester can replace 80 to 100 manual workers. As estimated by UNICA, in the state of São Paulo alone, 82,200 manual sugarcane field workers face potential job loss (Guilhoto et al. 2002; Duarte et al. 2013; Moraes et al. 2015). On the other hand, mechanical harvesting is expected to improve working conditions, average salary and gender equity in the sugarcane sector in Brazil. These findings are consistent with those of Souza et al. (2016), who concluded that manual harvesting leads to creation of more employment while mechanical harvesting results in a lower level of occupational accidents, higher average wages and more participation of female workers.

3.3 Comparing the results of SHDB and content analysis 
SHDB and content analysis identified 15 and 12 social hotspots respectively in the sugarcane sector in Brazil, with $60 \%$ of the social hotspots identified in SHDB confirmed by the content analysis. This confirms that SHDB is a useful tool to identify social risks associated with a country-sector. However, at the moment, SHDB has limited ability to distinguish between social impacts arising in different sectors in the same country or associated with different production routes within the same country-sector. Content analysis gives a much richer picture of the impacts and the consequences of changes in the product system. In this specific example, there are large differences in social impacts between manual and mechanical harvesting (see Section 3.2), but these differences are not captured in SHDB in its current form. However, in future studies, with better input-output data and sectoral impact inventories, the product systems and impacts of manual and mechanical harvesting may be differentiated.

The limitations of SHDB can be overcome by combining it with content analysis. Unlike SHDB, which only assesses negative impacts, content analysis is able to identify positive impacts such as, in this case, the industrial association's endeavor to promote public commitment to sustainability issues and the impacts of increasing mechanical harvesting in increasing average salaries. In addition, content analysis can facilitate data collection for foreground processes and provide more comprehensive understanding of the sectoral context, enabling better judgements on the status and cause of social impacts. Content analysis can further benefit the design of approaches and materials to collect site-specific primary data, for example via questionnaires and interviews, which can eventually improve SLCA's accessibility (Grubert 2018). However, it is worth noting that obtaining in-depth information through content analysis is at the cost of requiring more time for gathering and analyzing literature.

\section{Conclusions}

This study reports a screening S-LCA to identify the social hotspots related to sugarcane production in Brazil. Social impacts are modelled using the Social Hotspots Database (SHDB) and analyzed further through content analysis of relevant literature. The sugarcane sector itself is the dominant country-sector contributing to the overall social impacts of the sugarcane life-cycle in Brazil, with other sectors representing nugatory contributions to working hours and hence to social impacts. On the impact category level, the SHDB identifies "health and safety" and "labour rights and decent work" as the most significant impact categories. Social hotspots of sugarcane sector in Brazil identified in both SHDB and content analysis, nine in total, include "health and safety", "fair salary", "social benefits and social security", "access to material resources", "delocalization and migration", "forced labour", "safe and healthy living conditions", "freedom of association and collective bargaining", and "equal opportunity and discrimination". Comparing the results of both approaches shows that SHDB is effective for identifying social impacts at the country level; however, it is less effective at the sector level due to the aggregation of the data. Integrated use of content analysis with SHDB can improve the quality of inventory data for foreground processes by considering the magnitude and cause of the social impacts. Content analysis provides an enriched picture of the impacts of a product system and enables alternative production routes within the same sector to be differentiated. Both positive and negative impacts can be identified by content analysis, which are not included in SHDB. When applicable, the improved screening SLCA results applying SHDB and content analysis benefit the design of the 
approaches and materials to be used in site-specific data collection. Considering these advantages, we recommend use of content analysis in combination with SHDB to improve the results of a screening S-LCA.

Acknowledgements

This research was carried out in the framework of the Energy for Sustainability Initiative (EfS) of the University of Coimbra (UC), Portugal, and the MIT-Portugal Program, and was co-funded by FEDER and Fundação para a Ciência e Tecnologia (FCT) through project "Sustainability assessment of bioenergy systems: a life cycle multicriteria decision-support approach, including land use change", POCI-01-0145FEDER-016765 (PTDC/AAGMAA/6234/2014). Chongyang Du acknowledges financial support from FCT, through grant SFRH/BD/51948/2012. Roland Clift acknowledges the EfS Initiative for supporting his visits to the UC.

References

Amaral LF (2011) The Brazilian sugarcane sector experience : promoting sustainability with adequate tools. In: Euroclima Project Seminar CTBE-Unicamp-JRC. http://iet.jrc.ec.europa.eu/remea/sites/remea/files/files/documents/events/amaral_sugarcane_0.pdf. Accessed 18 October 2017

Arbex MA, Bohm GM, Saldiva PHN et al (2000) Assessment of the effects of sugar cane plantation burning on daily counts of inhalation therapy. J Air Waste Manage Assoc 50:1745-1749. doi: $10.1080 / 10473289.2000 .10464211$

Arbex MA, Martins LC, Oliveira RC et al (2007) Air pollution from biomass burning and asthma hospital admissions in a sugar cane plantation area in Brazil. J Epidemiol Community Heal 61:395-400. doi: $10.1136 /$ jech.2005.044743

Arcese G, Lucchetti MC, Massa I, Valente C (2018) State of the art in S-LCA: integrating literature review and automatic text analysis. Int J Life Cycle Assess 23(3):394-405.

Benoit-Norris C, Aulisio D, Norris GA (2012) Working with the Social Hotspots Database - methodology and findings from 7 social scoping assessments. In: 19th CIRP International Conference on Life Cycle Engineering. doi: 10.1007/978-3-642-29069-5_98

Benoit-Norris C, Aulisio D, Norris GA (2012) Identifying social impacts in product supply chains: overview and application of the Social Hotspot Database. Sustainability 4:1946-1965. doi:10.3390/su4091946

Benoit-Norris C, Norris GA, Aulisio D (2013) Social hotspots database supporting documentation. http://socialhotspot.org/. Accessed 18 October 2017 
BONSUCRO (2017) https://www.bonsucro.com/en/. Accessed 18 October 2017

Chaddad FR (2010) UNICA : Challenges to deliver sustainability in the Brazilian sugarcane industry. Int Food Agribus Manag Rev 13:173-192.

Ciroth A, Franze J (2011) LCA of an Ecolabeled Notebook: consideration of social and environmental impacts along the entire life cycle. Federal Public Planning Service Sustainable Development. https://www.greendelta.com/wp-content/uploads/2017/03/LCA_laptop_final.pdf Accessed 01 November 2017

CTC - Centro de Tecnologia Canavieira (2014) http://www.ctcanavieira.com.br/index.html. Accessed 18 October 2017

Di Cesare S, Silveri F, Sala S, Petti L (2018) Positive impacts in social life cycle assessment: state of the art and the way forward. Int J Life Cycle Assess 23(3):406-421. doi:10.1007/s11367-016-1169-7

Dreyer L, Hauschild M, Schierbeck J (2006) A framework for Social Life Cycle Impact Assessment. Int J Life Cycle Assess 11:88. doi:10.1065/lca2005.08.223

Du C, Kulay L, Cavalett O, Dias L, Freire F (2017). Life cycle assessment addressing health effects of particulate matter of mechanical versus manual sugarcane harvesting in Brazil. Int J Life Cycle Assess. doi: 10.1007/s11367017-1334-7

Duarte CG, Gaudreau K, Gibson RB, Malheiros TF (2013) Sustainability assessment of sugarcane-ethanol production in Brazil: A case study of a sugarcane mill in São Paulo state. Ecol Indic 30:119-129. doi: 10.1016/j.ecolind.2013.02.011

Ekener E, Hansson J, Gustavsson M (2016) Addressing positive impacts in social LCA - discussing current and new approaches exemplified by the case of vehicle fuels. Int J Life Cycle Assess. doi: 10.1007/s11367-016-1058-0

Ekener-Petersen E, Höglund J, Finnveden G (2014) Screening potential social impacts of fossil fuels and biofuels for vehicles. Energy Policy 73:416-426. doi: 10.1016/j.enpol.2014.05.034

ELLA (2012) From manual to mechanical harvesting : reducing environmental impacts and increasing cogeneration potential. https://www.gov.uk/dfid-research-outputs/ella-policy-brief-from-manual-to-mechanical-harvestingreducing-environmental-impacts-and-increasing-cogeneration-potential. Accessed 18 October 2017

Food and Agricultural Organization of the United Nations - FAO (2016) http://faostat.fao.org/beta/en/. Accessed 18 October 2017

Garrido SR, Parent J, Beaulieu L, Revéret JP (2016) A literature review of type I S-LCA-making the logic underlying methodological choices explicit. Int J Life Cycle Assess. doi: 10.1007/s11367-016-1067-z

Global Reporting Initiative (2017) https://www.globalreporting.org/. Accessed 18 October 2017

Global Trade Analysis Project (2017) https://www.gtap.agecon.purdue.edu/databases/v9/. Accessed 18 October 2017

Goldemberg J, Coelho ST, Guardabassi P (2008) The sustainability of ethanol production from sugarcane. Energy Policy 36:2086-2097. doi: 10.1016/j.enpol.2008.02.028

Grubert E (2018) Rigor in social life cycle assessment: improving the scientific grounding of SLCA. Int J Life Cycle Assess 23:481-491. doi:10.1007/s11367-016-1117-6 
Guilhoto JJM, Barros ALM, Marjotta-Maistro MC, Istake M (2002) Mechanization process of the sugar cane harvest and its direct and indirect impact over the employment in Brazil and in its 5 macro regions. https://mpra.ub.uni-muenchen.de/38070/1/MPRA_paper_38070.pdf. Accessed 18 October 2017

Hall J, Matos S, Severino L, Beltrao N (2009) Brazilian biofuels and social exclusion : established and concentrated ethanol versus emerging and dispersed biodiesel. J Clean Prod 17:S77-S85. doi: 10.1016/j.jclepro.2009.01.003

Hermele $\mathrm{K}$ (2011a) Regulating sugarcane cultivation in Brazil. https://nishat2013.files.wordpress.com/2013/11/regulating-sugarcane-cultivation-in-brazil-book.pdf. Accessed 18 October 2017

Hermele K (2011b) Sustainable agrofuels, land use change \& certification schemes. In: IEA Bioenergy workshop: quantifying and managing land use effects of bioenergy. http://www.ieabioenergy-task38.org/Hermele__Sustainable_agro-fuels.pdf. Accessed 18 October 2017

Junior LCS, Ramos EMC, Toledo AC, Ceccato ADF et al (2012) Assessment of health-related quality of life of sugarcane cutters in the pre-harvest and harvest periods. Rev Saúde Pública 46:1058-1065.

Lehtonen M (2010) Power, certification, and the social sustainability of Brazilian bioethanol: views from Brazil. In: Berlin Conference on the Human Dimensions of Global Environmental Change. http://edocs.fuberlin.de/docs/servlets/MCRFileNodeServlet/FUDOCS_derivate_000000001364/Lehtonen-

Biofuel_certification_as_a_tool_for_environmental_and_social_sustainability-459.pdf?hosts=. Accessed 18 October 2017

Luz VG, Filho HRC, Silva AJN et al (2012) Migrant labor and wear-out in manual sugarcane harvesting in São Paulo, Brazil. Cien Saude Colet 17:2831-2840. doi: 10.1590/S1413-81232012001000030

Macedo IC (2007) Sugar Cane's energy - Twelve studies on Brazilian sugar cane agribusiness and its sustainability. In: 2nd edn. Berlendis Editores Ltda., pp 194-225

Machado PG, Walter A, Picoli MC, João CG (2016) Potential impacts on local quality of life due to sugarcane expansion: a case study based on panel data analysis. Environ Dev Sustain. doi: 10.1007/s10668-016-9823-6

Martinelli LA, Filoso S (2008) Expansion of sugarcane ethanol production in Brazil: environmental and social challenges. Ecol Appl 18:885-898.

Martinelli LA, Garrett R, Ferraz S, Naylor R (2011) Sugar and ethanol production as a rural development strategy in Brazil: Evidence from the state of São Paulo. Agric Syst 104:419-428. doi: 10.1016/j.agsy.2011.01.006

Mayring P (2014) Qualitative content analysis: theoretical foundation, basic procedures and software solution. http://www.psychopen.eu/fileadmin/user_upload/books/mayring/ssoar-2014-mayring-

Qualitative_content_analysis_theoretical_foundation.pdf. Accessed 18 October 2017

McGrath S (2013) Fuelling global production networks with slave labour?: migrant sugar cane workers in the Brazilian ethanol GPN. Geoforum, 44: 32-43.

Moraes MA (2011) Lessons from Brazil. Nature 474:S25. doi: 10.1038/474S025a

Moraes MA (2007) Labor market indicators of the sugar cane agrosystem in Brazil. In: Ethanol Summit. http://www.mp.go.gov.br/nat_sucroalcooleiro/Documentos/palestras/summit/labor.pdf. Accessed 18 October 2017

Moraes MA (2008) Sugar Cane Sector in Brazil: labor indicators and migration. In: ESALQ, University of São Paulo.https://migrationfiles.ucdavis.edu/uploads/cf/files/conference_may_2008/azanha-

sugar_cane_sector_in_brazil-labor_indicators_and_migration.pdf. Accessed 18 October 2017 
Moraes MA, Oliveira FCR, Diaz-Chavez RA (2015) Socio-economic impacts of Brazilian sugarcane industry. Environmental Development. doi: 10.1016/j.envdev.2015.06.010.

NVivo (2017) http://www.qsrinternational.com/nvivo-product. Accessed 18 October 2017

Petti L, Serreli M, Di Cesare S (2018) Systematic literature review in social life cycle assessment. Int J Life Cycle Assess 23:422-431. doi:10.1007/s11367-016-1135-4

Priuli RMA, Moraes MS, Chiaravalloti RM (2014) The impact of stress on the health of sugar cane cutters. Rev Saúde Pública 48:1-6. doi: 10.1590/S0034-8910.2014048004798

Ramirez PKS, Petti L, Brones F, Ugaya CML (2016) Subcategory assessment method for social life cycle assessment. Part 2: application in Natura's cocoa soap. Int J Life Cycle Assess 21:106-117. doi: 10.1007/s11367015-0964-x

Rocha FLR, Marziale MHP, Hong OS (2010) Work and health conditions of sugar cane workers in Brazil. Rev Esc Enferm USP 44:974-979.

Russell E, Lee, J, Clift R (2018) Can the SDGs provide a basis for supply chain decisions in the construction sector? Sustainability in press.

Santos UP, Zanetta DMT, Terra-Filho M, Burdmann EA (2015) Burnt sugarcane harvesting is associated with acute renal dysfunction. Kidney Int 87:792-799. doi: 10.1038/ki.2014.306

Smeets E, Junginger M, Faaij A et al (2008) Sustainability of Brazilian ethanol - an assessment of the possibilities of certified production. Biomass and Bioenergy. 32: 781-813.

Souza A, Watanabe MDB, Cavalett $O$ et al (2016) Social life cycle assessment of first and second-generation ethanol production technologies in Brazil. Int J Life Cycle Assess. doi: 10.1007/s11367-016-1112-y

Stemler S, Bebell D (1999) An empirical approach to understanding and analyzing the mission statements of selected educational institutions. The Annual Conference of the New England Educational Research Organization, April 9, 1999. https://files.eric.ed.gov/fulltext/ED442202.pdf. Accessed 17 February 2018

Stemler S (2001) An overview of content analysis. http://pareonline.net/getvn.asp?v=7\&n=17. Accessed 18 October 2017

UNEP/SETAC (2009) Guidelines for Social Life Cycle Assessment. http://www.unep.fr/shared/publications/pdf/dtix1164xpa-guidelines_S-LCA.pdf. Accessed 18 October 2017

UNEP/SETAC (2013) The Methodological Sheets for Sub-categories in Social Life Cycle Assessment. http://www.lifecycleinitiative.org/wp-content/uploads/2013/11/S-LCA_methodological_sheets_11.11.13.pdf.

Accessed 18 October 2017

UNICA (2010) UNICA Sustainability Report 2010. http://www.unica.com.br/sustainability-reporting/. Accessed 18 October 2017

UNICA (2017) UNICADATA project. http://www.unicadata.com.br/. Accessed 18 October 2017

Uriarte M, Yackulic CB, Cooper T et al (2009) Expansion of sugarcane production in São Paulo, Brazil: Implications for fire occurrence and respiratory health. Agric Ecosyst Environ 132:48-56. doi: 10.1016/j.agee.2009.02.018

Viana KRO, Perez R (2013) Survey of sugarcane industry in Minas Gerais, Brazil: Focus on sustainability. Biomass and Bioenergy 58:149-157. doi: 10.1016/j.biombioe.2013.08.006 
Walter A, Dolzan P, Quilodran O et al (2011) Sustainability assessment of bio-ethanol production in Brazil considering land use change, GHG emissions and socio-economic aspects. Energy Policy 39:5703-5716

Xavier CV, Pitta FT, Mendoça ML (2011) A monopoly in ethanol production in Brazil: The Cosan-Shell merger. http://www.social.org.br/ethanol_monopoly_brazil.pdf. Accessed 15 November 2016

Zamani B, Sandin G, Svanström M, Peters GM (2016) Hotspot identification in the clothing industry using social life cycle assessment - opportunities and challenges of input-output modelling. Int J Life Cycle Assess. doi: $10.1007 / \mathrm{s} 11367-016-1113-\mathrm{x}$

Zhang Y, Wildemuth BM (2009) Qualitative Analysis of Content. In: Applications of Social Research Methods to Questions in Information and Library Science. Westport, CT: Libraries Unlimited., pp 308-319 